\title{
Cryotherapy and ankle motion in chronic venous disorders
}

\author{
Teresa J. Kelechi ${ }^{1}$, Martina Mueller ${ }^{1}$, Jane G. Zapka², Dana E. King ${ }^{3}$ \\ ${ }^{1}$ College of Nursing, Medical University of South Carolina, Charleston, USA \\ ${ }^{2}$ Biostatistics and Bioepidemiology, Medical University of South Carolina, Charleston, USA \\ ${ }^{3}$ Department of Family Medicine, West Virginia University, Morgantown, USA \\ Email: kelechti@musc.edu, muellerm@musc.edu, zapka@musc.edu, kingdana@,wvuhealthcare.com
}

Received 5 September 2012; revised 12 October 2012; accepted 14 November 2012

\begin{abstract}
This study compared ankle range of motion (AROM) including dorsiflexion, plantar flexion, inversion and eversion, and venous refill time (VRT) in leg skin inflamed by venous disorders, before and after a new cryotherapy ulcer prevention treatment. Fifty-sevenindividuals participated in the randomized clinical trial; 28 in the experimental group and 29 received usual care only. Results revealed no statistically significant differences between the experimental and usual care groups although AROM measures in the experimental group showed a consistent, non-clinically relevant decrease compared to the usual care group except for dorsiflexion. Within treatment group comparisons of VRT results showed a statistically significant increase in both dorsiflexion and plantar flexion for patients with severe VRT in the experimental group $(6.9 \pm 6.8 ; p=0.002$ and $5.8 \pm 12.6 ; p=0.02$, respectively). Cryotherapy did not further restrict already compromised AROM, and in some cases, there were minor improvements.
\end{abstract}

Keywords: Cryotherapy; Ankle Range of Motion; Chronic Venous Disorders; Chronic Venous Insufficiency; Venous Refill Time

\section{INTRODUCTION}

Ankle range of motion (AROM) is impaired in individuals with chronic venous disorders and was the focus of this study to determine the efficacy of using a cryotherapy gel wrap on leg skin circulation where skin damage and healed venous ulcers were present. The randomized clinical trial compared a usual care protocol to a specially-designed cooling gel wrap applied to the lower leg and ankle area combined with usual care for 30 minutes a day for one month. The aim was to determine if cooling the skin and ankle area further restricted AROM. The long-term objective is to demonstrate that cooling the skin could prevent new leg ulcers in damaged skin and reduce the recurrence of ulcer in individuals with a his- tory of ulcers associated with chronic venous disorders.

It is estimated that 7 million people in the United States are affected by chronic venous disorders such as venous insufficiency and venous disease [1]. These disorders impact the structure and function of the lower extremity vasculature, typically involving the ankle, where range of motion is impaired, and the skin becomes damaged. New ulcers develop in the skin around or just above the ankle in approximately 600,000 patients each year [2]. An additional 2.5 to 3 million suffer with chronic, slow-to-heal lower leg ulcers at any given time [3]. The inability to effectively prevent and treat chronic venous disorders contributes substantially to the high rate of chronicity and disease sequelae, including a lifetime of recurring leg ulcers.

The disease sequelae, in part, stems from sustained ambulatory venous hypertension, hypothesized to be the underlying pathological event that eventually results in the stretching of the vein pores. The stretching allows blood components to leak into the interstitial tissues, inciting an inflammatory reaction that causes skin damage and ulceration $[4,5]$. It is suggested that venous hypertension and deterioration of the calf muscle pump are related. Loss of AROM, particularly dorsiflexion, has been implicated as a potential factor contributing to ineffective calf muscle pumping, affecting between $32 \%$ and $70 \%$ of patients with venous insufficiency [6,7]. Studies have demonstrated a relationship between ineffective calf muscle function and impaired AROM [8,9], where the ability of the calf muscle to effectively pump or eject blood out of the lower leg is reduced, resulting in blood stasis in the large and perforating vessels. In particular, the ankle skin below the calf is affected due to the backup of blood into abundant cutaneous blood vessels located in this region of the leg. Regardless of whether calf muscle dysfunction is present, studies show a strong relationship between chronic venous disorders and impaired AROM [6,10-12].

A host of other factors play a role in patients with chronic venous disorders and impaired AROM. Comorbid conditions such as obesity [13,14], diabetes [15], 
and arthritis [16,17] have been found to be associated with ankle stiffness, reduced dorsiflexion range of motion, and leg ulcers. Treatment of edema associated with chronic venous disorders with compression is an additional confounding factor that likely influences AROM. Multi-layer high compression (40 - $50 \mathrm{~mm} \mathrm{Hg}$ ), applied from the base of the toes to just below the knee, is the mainstay of leg ulcer management. However, high compression restricts ankle joint movement [18]. Finally, pain, a common accompaniment of leg ulcers, hampers AROM because patients find that even small movements in their feet and lower legs can cause discomfort and achiness, thus leading to lack of physical activity and stiffness of joints [19]. Due to a multitude of factors, intervention strategies to ameliorate impaired AROM remain elusive.

\section{METHOD}

This study builds on a larger randomized controlled trial to test a cryotherapy (cooling) treatment on leg skin around the ankle in patients with severe venous insufficiency [20]. The purpose of this study arm, also a randomized trial, was to examine the relationships among AROM (specifically dorsiflexion, plantar flexion, inversion and eversion), venous refill time (VRT) of the cutaneous vessels above the medial malleolus of the most severely affected leg (an outcome variable for the primary study), and ankle circumference (AC), before and after the cooling intervention. We also evaluated AROM by categories of body mass index (BMI) and $\mathrm{AC}$, recognizing that higher BMIs and larger ACs could negatively impact AROM. We hypothesized that there would be no differences in AROM for VRT and AC after the cooling treatment. The rationale for this investigation was that using ice or cyrotherapy, a widely accepted and practiced treatment for inflammation associated with acute soft tissue injury, could be applied to chronically-inflamed-skin to reduce swelling, and improve circulatory function [21]. However, little is known about the effects of cooling chronically inflamed skin and joint mobility. We did not want to further restrict AROM with the cooling therapy, however, we were interested to learn if cooling the ankle region affected by chronic venous disorders might have a positive effect, such as improving AROM.

\subsection{Sample}

In accordance with Institutional Review Board study approval, sixty patients from wound clinics, primary care practices, and the general community were recruited, consented, and enrolled in the one-month trial conducted 2008-2010. If eligible after screening, patients were enrolled and randomized to receive the cooling treatment coupled with usual care $(\mathrm{n}=30)$ or usual care only $(\mathrm{n}=$ 30 ). Inclusion criteria were age 21 years or older, diagnosis of chronic venous disorders, ankle brachial index (ABI) $0.8-1.3 \mathrm{~mm} \mathrm{Hg}$ (arterial sufficiency) measured with a blood pressure cuff and Doppler, Clinical-Etiological-Anatomical-Pathological (CEAP) [22] stages C4 (skin damage) or C5 (history of venous leg ulcers), intact skin sensation measured at the plantar surface of the foot with a 10 gram monofilament, intact thermal sensation measured over the dorsum of the foot with a thermal sensor (Tip Therm ${ }^{\circledR}$, Bailey Instruments LTD, Salford Quays, England), and venous refill time (VRT) $\leq 25$ seconds (indicates venous insufficiency) measured with a handheld venous photoplethysmograph (PPG) (Huntleigh, Eatontown NJ). Subjects also had to have a working freezer to store the cooling wrap and agree to wear compression during waking hours.

Exclusion criteria were having an open ulcer, surgical procedures performed on the affected leg in past one year (which can affect venous circulation/cause edema), impaired cognitive status (cannot perform procedures), chronic inflammatory and vascular conditions such as Lupus erythematosus, lymphedema, Raynaud's phenomena or disease, rheumatoid arthritis, scleroderma, end stage renal disease, chronic obstructive pulmonary disease, chronic regional pain syndrome, multiple sclerosis, hypersensitivity to cold, or patients on chemotherapy (blood flow of the skin or AROM might be impacted).

\subsection{Procedures}

The experimental conditions consisted of a daily 30minute treatment with a cooling gel wrap (Southwest Technologies, Kansas City, MO) placed over the lowest portion of the leg around the ankle of the most affected leg. The experimental group patients also engaged in usual care that consisted of the wearing of compression stockings (except during treatment and at night) and 30 minutes of leg elevation daily. Patients randomized to the usual care (only) group wore compression stockings (except during treatment and at night) and elevated their legs for 30 minutes without using the cooling wrap. Both groups measured the lower leg skin temperature with a long handled infrared thermometer (Temp Touch, Diabetica Solutions, San Antonio, TX) at an inked spot, measured $5 \mathrm{~cm}$ above the medial malleolus, before and after each treatment, and recorded it on a log sheet. This inked area was the location on the lower leg chiefly affected by venous insufficiency with damaged skin. The purpose of temperature monitoring was to prevent excessive cooling or frostbite and to track adherence. The goal was to have skin temperature at the ankle reduced by $10^{\circ} \mathrm{C}$ to $15^{\circ} \mathrm{C}$ from baseline, in contrast to the standard $25^{\circ} \mathrm{C}$ used to reduce metabolic activity from acute injury. If the temperature was outside of the range, patients were 
to call the study nurse.

\subsection{Study Endpoints and Instruments}

\subsubsection{AROM}

Plantar flexion and dorsiflexion were measured with a standard $12 \frac{1}{2}$ inch plastic goniometer while inversion and eversion were measured with an 8 inch plastic goniometer (Sammons Preston Rolyan Company, Cedarburg, WI). Measurements were reported in degrees. Normal values for this study were: Ankle plantar flexion (movement downward) $\sim 50^{\circ}$; ankle dorsiflexion (movement upward) $\sim 20^{\circ}$; foot inversion (turned inward) $\sim 35^{\circ}$; and foot eversion (turned outward) $\sim 20^{\circ}$ [23]. One study nurse, using the same goniometer for each measurement, collected all study data. Trained by a physical therapist (PT) using the procedure described by Norkin and White [23], she began AROM measurements in the study after she achieved 100\% interrater agreement with the PT's goniometric readings. The goniometer used in this study has intrarater reliability using intraclass correlation coefficient (ICC) of 0.92 for dorsiflexion and 0.96 for plantar flexion [24]. ICC for inversion and eversion is reported as high as 0.90 and 0.80 respectively [25].

\subsubsection{Venous Refill Time}

Venous refill time (VRT), measured with a hand-held photoplethysmograph (PPG), detects venous reflux, an indicator of venous insufficiency. VRT is defined as the time it takes blood to refill the microcirculation after a series of 10 foot dorsiflexions, toes lifted up with heel firmly planted, conducted with the patient in the seated position, knees bent, and feet flat on the floor. Dorsiflexions cause the calf muscle to contract and empty the veins. The time taken to refill the small cutaneous vessels at the ankle was then measured with a probe placed five $\mathrm{cm}$ above the medial malleolus, and recorded in seconds with the PPG (Huntleigh, Eatontown NJ). Findings of $\leq 25$ seconds indicate that the calf pump is ineffective in ejecting blood and thus, the engorged larger vessels quickly refill the smaller vessels, an abnormal finding [26]. The PPG used in this study has an intrarater reliability using ICC reported as high as 0.98 [27].

\subsubsection{Ankle Circumference}

A standard tape measure was used to measure the circumference of the ankle, five $\mathrm{cm}$ above the medial malleolus, at the same location where VRT was measured, while the patient was in the standing position. This measurement provided an estimation of the presence of edema. Both legs were measured for comparison.

\subsection{Study Power}

As AROM measures were secondary outcomes for the larger, randomized study, the study was not adequately powered to detect statistically significant differences in AROM measurements between the treatment and usual care groups. However, for the between group (cooling versus usual care) comparison of AROM change from baseline to follow-up, based on a two-sided pooled t-test comparison with level of significance $\alpha=0.05$, there was approximately $80 \%$ power to detect a difference of 0.8 standard deviation (SD) units.

\subsection{Data Analysis}

The study sample was described in terms of demographic and clinical characteristics using measures of central tendency or frequency distributions. For comparisons between the treatment groups, pooled t-test or chi-square tests were used. AROM means were compared between baseline and follow-up within treatment groups (cooling and usual care) using Wilcoxon Signed Rank tests, while AROM means were compared between VRT levels using Kruskal-Wallis tests. Similarly, means and SDs for AROM measures were reported for two levels of ankle circumference (AC): $25 \mathrm{~cm}$ or less versus more than $25 \mathrm{~cm}$ within the treatment groups. Within each treatment group AROM mean change from baseline to follow-up was compared using pooled t-tests and AROM differences in means at baseline and at follow-up between the AC levels were compared using Wilcoxon Signed Rank tests. In addition, adjusted AROM means from general linear models (GLM) are reported using change in AROM measure from baseline to follow-up as dependent measures, treatment group as primary independent variable of interest adjusted for baseline AROM measurement and individually for number of previous ulcers, VRT level, ankle circumference, and BMI. Mean differences in adjusted AROM measure between the groups are reported along with $95 \%$ confidence intervals. Further, distributions of mean eversion-inversion flexion and mean dorsi-plantar flexion at baseline by BMI category (normal: 18.5 - 24.9; overweight: 25.0 - 29.9; obese: 30 - 39.9; morbidly obese: $>40.0)$ are shown.

\section{RESULTS}

Of the 60 patients consented and enrolled, a total of 57 completed the study $(\mathrm{n}=28$ experimental and $\mathrm{n}=29$ usual care). One patient dropped out due to personal reasons, one was lost to follow-up, and one was withdrawn due to sustaining a traumatic wound to the unaffected leg over which she could not wear compression stockings. Overall the mean age was $61.2 \pm 12.7 ; 70.2 \%$ were female, $50.9 \%$ were African-American or Black, and $47.4 \%$ were White. The majority of participants were 
obese or morbidly obese (see Table 1). At the start of the study, individuals randomized to receive the coolingtreatment had statistically significantly smaller ankle circumferences $(24.9 \pm 3.6$ versus $27.3 \pm 4.9 ; p=0.05)$. Participants randomized to cooling started out with statistically significantly higher plantar flexion compared to the usual care group $(26.7 \pm 7.9$ vs. $22.2 \pm 7.5, p=0.03)$. All participants had considerably lower AROM (dorsiflexion, plantar flexion, inversion and eversion) compared to normal values.

\section{Study Endpoints}

Table 2 reports mean AROM measures from GLMs adjusted for putative predictor variables. After adjustment, there were no statistically significant differences between the experimental and usual care groups although AROM measures in the experimental group showed a consistent decrease compared to the usual care group except for dorsiflexion.
Within treatment group comparisons of VRT levels results showed a statistically significant increase in both dorsiflexion and plantar flexion for patients with severe VRT levels in the cooling group $\left(6.9^{\circ} \pm 6.8 ; p=0.002\right.$ and $5.8^{\circ} \pm 12.6 ; p=0.02$, respectively). No further statistically significant differences in AROM measures of dorsiflexion, plantar flexion, inversion, or eversion at baseline and after the 30-day study period for VRT were found (Table 3). Furthermore, no statistically significant differences were noted when between-group analyses were performed for VRT levels.

For the ankle circumference category $25 \mathrm{~cm}$ or less a statistically significant increase in dorsiflexion was observed in the usual care group $\left(8.2^{\circ} \pm 6.4 ; p=0.002\right)$ while the increase was not significant in the cooling group $\left(3.5^{\circ} \pm 7.7 ; p=0.10\right)$. Though only marginally statistically significant in the cooling group, inversion was almost $5^{\circ}$ less after 30 days for patients with larger ankle circumferences compared to those with thinner

Table 1. Demographic and clinical characteristics at baseline $(n=57)$.

\begin{tabular}{|c|c|c|c|}
\hline & Cryotherapy $(n=28)$ & Usual care $(n=29)$ & $p$-value \\
\hline Age (mean [SD]) & $61.6(12.1)$ & $60.9(13.4)$ & 0.851 \\
\hline \multicolumn{4}{|l|}{ Age categories } \\
\hline $35-49$ years & $17.9 \%(5 / 28)$ & $17.2 \%(5 / 29)$ & \multirow{3}{*}{0.993} \\
\hline $50-69$ years & $53.6 \%(15 / 28)$ & $55.2 \%(16 / 29)$ & \\
\hline 70 years or older & $28.6 \%(8 / 28)$ & $27.6 \%(8 / 29)$ & \\
\hline Gender (Female) & $71.4 \%(20 / 28)$ & $69.0 \%(20 / 29)$ & 0.839 \\
\hline \multicolumn{4}{|l|}{ Race/ethnicity } \\
\hline White & $46.4 \%(13 / 28)$ & $48.3 \%(14 / 29)$ & \multirow{2}{*}{$0.992^{*}$} \\
\hline African-American & $50.0 \%(14 / 28)$ & $51.7 \%(15 / 29)$ & \\
\hline Latino & $3.6 \%(1 / 28)$ & 0 & \\
\hline \multicolumn{4}{|l|}{ Occupation } \\
\hline Professional & $42.9 \%(12 / 28)$ & $44.8 \%(13 / 29)$ & \multirow{3}{*}{0.562} \\
\hline Technical & $32.1 \%(9 / 28)$ & $20.7 \%(6 / 29)$ & \\
\hline Labor & $25.0 \%(7 / 28)$ & $34.5 \%(10 / 29)$ & \\
\hline Residence (Urban) & $78.6 \%(22 / 28)$ & $79.3 \%(23 / 29)$ & 0.946 \\
\hline BMI (mean [SD]) & $36.8(11.5)$ & $39.3(12.1)$ & 0.439 \\
\hline \multicolumn{4}{|l|}{ BMI categories } \\
\hline Normal (18.5 - 24.9) & $10.7 \%(3 / 28)$ & $17.2 \%(5 / 29)$ & \multirow{4}{*}{$0.341^{* *}$} \\
\hline Overweight (25.0 - 29.9) & $21.4 \%(6 / 28)$ & $10.3 \%(3 / 29)$ & \\
\hline Obese $(30.0-39.9)$ & $32.1 \%(9 / 28)$ & $24.1 \%(7 / 29)$ & \\
\hline Morbidly obese $(\geq 40.0)$ & $35.7 \%(10 / 28)$ & $48.3 \%(14 / 29)$ & \\
\hline Calf circumference (mean [SD]) & $42.5(7.0)$ & $44.0(6.3)$ & 0.419 \\
\hline Ankle circumference (mean [SD]) & $24.9(3.6)$ & $27.3(4.9)$ & 0.035 \\
\hline Doppler VRT (mean [SD]) & $18.1(13.8)$ & $12.7(12.6)$ & 0.110 \\
\hline Compression stockings (Yes) & $75.0 \%(21 / 28)$ & $55.2 \%(16 / 29)$ & 0.117 \\
\hline
\end{tabular}

"From Chi-square test for White vs. African-American only; ${ }^{* *}$ From Chi-square test for Normal or Overweight vs. Obese vs. Morbidly obese. 
Table 2. Adjusted means (and standard errors) ${ }^{*}$ from general linear models with change in AROM measures from baseline by treatment group.

\begin{tabular}{|c|c|c|c|c|c|}
\hline Eversion & Cryotherapy & Usual care & Difference & $95 \%$ CI & $p$-value \\
\hline \#previous ulcers & $1.35(1.47)$ & $-1.30(1.44)$ & 2.65 & $-1.52 ; 6.82$ & 0.208 \\
\hline VRT level & $1.53(1.49)$ & $-1.88(1.50)$ & 3.42 & $-0.89 ; 7.72$ & 0.117 \\
\hline Ankle circumference & $1.61(1.46)$ & $-1.60(1.44)$ & 3.22 & $-0.99 ; 7.42$ & 0.131 \\
\hline BMI & $1.34(1.47)$ & $-1.29(1.44)$ & 2.63 & $-1.55 ; 6.81$ & 0.213 \\
\hline Inversion & Cryotherapy & Usual care & Difference & $95 \% \mathrm{CI}$ & $p$-value \\
\hline \#previous ulcers & $1.95(1.52)$ & $-1.19(1.49)$ & 3.14 & $-1.16 ; 7.44$ & 0.149 \\
\hline VRT level & $2.57(1.51)$ & $-1.37(1.52)$ & 3.94 & $-0.40 ; 8.28$ & 0.074 \\
\hline Ankle circumference & $2.28(1.48)$ & $-1.58(1.46)$ & 3.86 & $-0.39 ; 8.11$ & 0.074 \\
\hline BMI & $1.93(1.52)$ & $-1.17(1.49)$ & 3.11 & $-1.19 ; 7.40$ & 0.153 \\
\hline Dorsiflexion & Cryotherapy & Usual care & Difference & $95 \% \mathrm{CI}$ & $p$-value \\
\hline \#previous ulcers & $-3.12(1.41)$ & $-3.43(1.39)$ & 0.31 & $-3.68 ; 4.30$ & 0.877 \\
\hline VRT level & $-2.94(1.47)$ & $-3.38(1.50)$ & 0.43 & $-3.80 ; 4.66$ & 0.839 \\
\hline Ankle circumference & $-2.63(1.40)$ & $-3.98(1.37)$ & 1.35 & $-2.63 ; 5.34$ & 0.498 \\
\hline BMI & $-3.04(1.43)$ & $-3.51(1.40)$ & 0.48 & $-3.56 ; 4.51$ & 0.814 \\
\hline Plantar flexion & Cryotherapy & Usual care & Difference & $95 \%$ CI & $p$-value \\
\hline \#previous ulcers & $0.43(1.70)$ & $-1.52(1.66)$ & 1.95 & $-2.92 ; 6.81$ & 0.426 \\
\hline VRT level & $0.30(1.73)$ & $-0.98(1.76)$ & 1.28 & $-3.79 ; 6.35$ & 0.615 \\
\hline Ankle circumference & $0.62(1.71)$ & $-1.73(1.68)$ & 2.36 & $-2.59 ; 7.30$ & 0.344 \\
\hline BMI & $0.42(1.69)$ & $-1.51(1.66)$ & 1.94 & $-2.92 ; 6.79$ & 0.428 \\
\hline
\end{tabular}

*Adjusted for baseline AROM measure and individually for number of previous ulcers, VRT level, ankle circumference, BMI.

ankles $\left(17.7^{\circ} \pm 6.5\right.$ vs. $\left.24.4^{\circ} \pm 9.5 ; p=0.06\right)$. For plantar flexion in both $\mathrm{AC}$ groups, there was a non-statistically significant decrease after cooling. Study participants in the lower BMI groups tended to have higher AROM measurements compared to heavier participants.

\section{DISCUSSION}

Our patients having the severest stages of chronic venous disorders, CEAP 4 (skin damage) and 5 (history of ulcers) showed reduced AROM at the start of the study to about two-thirds below normal values. The cooling treatment group did not experience a further statistically significant reduction in AROM, the key finding of this study. While there was a statistically significant difference in dorsiflexion in the usual group with $\mathrm{AC}<25 \mathrm{~cm}$, these differences were not considered to be clinically important as this group was small $(\mathrm{n}=17)$ and had better AROM at the start of the study. While the primary aim of the study was to establish relationships among AROM, VRT, and $\mathrm{AC}$, before and after the cooling intervention, there was a concern that applying a cooling wrap to functionally compromised ankles could further reduce AROM. The hypothesis that there would be no differences in primary endpoints AROM, VRT, and AC after the cooling treatment within and between the treatment groups was only partially supported. Certain demographic and clinical factors may have had an impact on study findings.

\subsection{Demographic and Clinical Factors}

Ankle motion may be affected by certain demographic and clinical characteristics of the study population. The average age of the sample was 61 years. While age-related changes intuitively sound like a plausible explanation of reduced ankle motion, no research evidence suggests normal aging as a major cause: Study results are conflicting and data from recent research studies are not forthcoming. Findings from an older study of strength and range of motion published in 1986, measured in 20 normal men and 20 normal women divided equally into age groups (25 - 35 and 50 - 60 years), were not statistically significantly different between the age groups, genders or dominant and non-dominant limbs [28]. Another older study found age-related differences in ankle joint range of motion in 120 children (range 9 - 13 years), adolescents (14 - 16 years), and young adults (17 - 20 years) compared to data published for older subjects (21 79 years) [29]. Findings suggest a consistent downward trend in AROM from a maximum at 14 to 16 or 17 to 20 years to a minimum after age 60 years and this average decrement was greater for females than for males. The 
Table 3. Means (SD) of AROM measures by VRT level and study group.

\begin{tabular}{|c|c|c|c|c|}
\hline \multicolumn{5}{|l|}{ Cryotherapy } \\
\hline Eversion & Baseline & Follow-up & Difference & $p$-Value* \\
\hline VRT level: Marginal $(21-25) n=7$ & $27.9(8.6)$ & $27.1(7.6)$ & $-0.7(6.1)$ & 0.999 \\
\hline VRT level: Moderate $(10-20) n=13$ & $27.7(11.8)$ & $26.9(11.6)$ & $-0.8(10.6)$ & 0.857 \\
\hline VRT level: Severe $(<10) n=8$ & $33.8(8.4)$ & $28.1(10.3)$ & $-5.6(6.8)$ & 0.063 \\
\hline$p$-value ${ }^{* *}$ & 0.407 & 0.851 & 0.334 & \\
\hline \multicolumn{5}{|l|}{ Inversion } \\
\hline VRT level: Marginal $(21-25) n=7$ & $26.4(6.9)$ & $22.1(7.0)$ & $-4.3(6.7)$ & 0.250 \\
\hline VRT level: Moderate $(10-20) n=13$ & $23.1(10.3)$ & $22.7(10.5)$ & $-0.4(8.0)$ & 0.955 \\
\hline VRT level: Severe $(<10) n=8$ & $25.6(7.8)$ & $20.0(8.5)$ & $-5.6(10.8)$ & 0.250 \\
\hline$p$-value ${ }^{* *}$ & 0.611 & 0.828 & 0.298 & \\
\hline \multicolumn{5}{|l|}{ Dorsiflexion } \\
\hline VRT level: Marginal $(21-25) n=7$ & $8.6(8.5)$ & $12.9(7.0)$ & $4.3(9.8)$ & 0.313 \\
\hline VRT level: Moderate $(10-20) n=13$ & $11.2(8.7)$ & $15.4(8.5)$ & $4.2(7.3)$ & 0.089 \\
\hline VRT level: Severe $(<10) n=8$ & $11.9(8.8)$ & $10.0(10.0)$ & $-1.9(9.2)$ & 0.625 \\
\hline$p$-value ${ }^{* *}$ & 0.630 & 0.423 & 0.244 & \\
\hline \multicolumn{5}{|l|}{ Plantar flexion } \\
\hline VRT level: Marginal $(21-25) n=7$ & $25.0(5.8)$ & $22.9(6.4)$ & $-2.1(3.9)$ & 0.375 \\
\hline VRT level: Moderate $(10-20) n=13$ & $25.2(6.5)$ & $23.9(9.4)$ & $-1.3(10.6)$ & 0.572 \\
\hline VRT level: Severe $(<10) n=8$ & $30.6(10.8)$ & $26.4(6.4)$ & $-4.3(12.6)$ & 0.406 \\
\hline$p$-value ${ }^{* *}$ & 0.485 & 0.395 & 0.703 & \\
\hline \multicolumn{5}{|l|}{ Usual care } \\
\hline Eversion & Baseline & Follow-up & Difference & $p$-Value \\
\hline VRT level: Marginal $(21-25) n=6$ & $28.3(14.7)$ & $30.0(8.4)$ & $1.7(11.7)$ & 0.563 \\
\hline VRT level: Moderate $(10-20) n=7$ & $27.9(6.4)$ & $31.4(11.4)$ & $3.6(6.9)$ & 0.375 \\
\hline VRT level: Severe $(<10) n=16$ & $23.1(7.9)$ & $24.7(8.1)$ & $1.6(7.7)$ & 0.391 \\
\hline$p$-value ${ }^{* *}$ & 0.425 & 0.187 & 0.944 & \\
\hline \multicolumn{5}{|l|}{ Inversion } \\
\hline VRT level: Marginal $(21-25) n=6$ & $24.2(10.2)$ & $20.0(7.1)$ & $-4.2(3.8)$ & 0.125 \\
\hline VRT level: Moderate $(10-20) n=7$ & $24.3(9.8)$ & $29.3(9.8)$ & $5.0(11.9)$ & 0.438 \\
\hline VRT level: Severe $(<10) n=16$ & $19.1(10.4)$ & $22.2(7.7)$ & $3.1(10.3)$ & 0.223 \\
\hline$p$-value ${ }^{* *}$ & 0.428 & 0.205 & 0.127 & \\
\hline \multicolumn{5}{|l|}{ Dorsiflexion } \\
\hline VRT level: Marginal $(21-25) n=6$ & $12.5(6.9)$ & $11.7(8.2)$ & $-0.83(9.7)$ & 0.938 \\
\hline VRT level: Moderate $(10-20) n=7$ & $9.0(8.0)$ & $10.7(8.4)$ & $1.7(12.7)$ & 0.999 \\
\hline VRT level: Severe $(<10) n=16$ & $7.5(7.8)$ & $14.4(6.6)$ & $6.9(6.8)$ & 0.002 \\
\hline$p$-value ${ }^{* *}$ & 0.359 & 0.505 & 0.157 & \\
\hline \multicolumn{5}{|l|}{ Plantar flexion } \\
\hline VRT level: Marginal $(21-25) n=6$ & $21.7(4.1)$ & $25.0(11.4)$ & $3.3(8.8)$ & 0.500 \\
\hline VRT level: Moderate $(10-20) n=7$ & $25.7(8.9)$ & $23.6 \quad(6.3)$ & $-2.1(11.1)$ & 0.688 \\
\hline VRT level: Severe $(<10) n=16$ & $20.9(7.8)$ & $26.8(10.3)$ & $5.8(12.6)$ & 0.022 \\
\hline$p$-value ${ }^{* *}$ & 0.261 & 0.913 & 0.185 & \\
\hline
\end{tabular}

*From wilcoxon signed rank test; ${ }^{* *}$ From kruskal-wallis test. 
study conclusion provides limited support for the contention that age-related and gender differences in AROM exist.

The relationship between a clinical factor, obesity (BMI $>30 \mathrm{~kg} / \mathrm{m}^{2}$ ) and AROM is also poorly documented and evidence is conflicting. In a study of 20 obese and 20 non-obese men, there were no statistically significant differences between the two groups for plantar flexion and dorsiflexion [3]. However, findings from a study of 14 obese participants (six men, eight women) with BMI $>30 \mathrm{~kg} / \mathrm{m}^{2}$ and 14 (six men, eight women) with normal BMI showed that plantar flexion of the obese group was significantly reduced compared to the normal group [30]. Dorsiflexion was not reported in this study.

\subsection{AROM and AC}

The only statistically significant finding in this study was a change in dorsiflexion, observed in the usual care group during leg elevation, when $\mathrm{AC}$ was $25 \mathrm{~cm}$ or less. This finding did not hold for the cooling treatment group. However, the patients in the cooling group with AC 25 $\mathrm{cm}$ or less had better dorsiflexion $\left(11.2^{\circ} \pm 9.4\right)$ at baseline compared to the control group $\left(7.3^{\circ} \pm 6.8\right)$ and both groups had similar results at the end of the study (treatment $=14.7^{\circ} \pm 8.4$; control $=15.5^{\circ} \pm 6.1$ ). Normal dorsiflexion is $\sim 20^{\circ}$. A possible explanation is that while patients with smaller $\mathrm{AC}$ were laying down with their legs elevated during the treatment, many reported that they often "automatically exercised" their ankles in circles and up and down while doing the treatment. This activity might have improved dorsiflexion over the four-week study. This same "exercise" phenomenon was not reported by participants with $\mathrm{AC}$ greater than $25 \mathrm{~cm}$. Those with larger AC might have found it too difficult to move the ankle due to stiffness or from having more edema present, limiting the movement of the ankle. We also recognize the statistically significant difference in AC between the two groups at the start of the study. The control group was larger by $2.4 \mathrm{~cm}$, which could possibly account for the lack of improvement in AROM.

Intact dorsiflexion is required to effectively pump the calf muscle to squeeze the veins and empty the vessels to eject blood up out the leg. When impaired, venous hypertension increases and insufficiency (blood is not moved out of the venous system) results. The associations of venous insufficiency and impaired AROM have been investigated in numerous studies. In a study of 32 limbs of 26 adult men, Back [6] showed that the more severe the skin damage in patients with varying degrees of severity of venous insufficiency, the greater the degree of loss of AROM. There were statistically significant differences in AROM in all three venous groups ( $p$-values $<0.001$ per group), rated using the Society for Vas- cular Surgery/International Society for Cardiovascular Surgery: class 1 or 2, $\mathrm{n}=9$ limbs; class 3 with healed ulceration, $\mathrm{n}=9$ limbs; class 4 with active ulceration, $\mathrm{n}=8$ limbs) compared to six normal controls (class 0 ). However AROM was most impaired in class 4 where there were severe clinical symptoms including skin inflammation and a history of leg ulcers. Similarly, Dix [11] studied 47 limbs of adults with varying degrees of venous insufficiency, staged by the Clinical-Etiology-Anatomical-Pathological (CEAP) Classification and 11 normal controls. Venous hypertensive limbs with the most severe stage of venous insufficiency, CEAP stage 6 (active ulcer) experienced the most pronounced reduction in plantar flexion and dorsiflexion. However, measurements of both plantar flexion and dorsiflexion showed that there were statistically significant differences in all limbs with CEAP stages $2-6$, compared to controls $p<0.004$. Cavalheri [10] showed that patients with the more severe stages of venous insufficiency, CEAP stages 5 (history of ulcer) and 6 (open leg ulcer) $(n=49)$ compared to CEAP stages $0-4(\mathrm{n}=140)$, experienced statistically significantly restricted AROM $p<0.05$. Panny [12] (2009) most recently conducted an investigation of 84 limbs in 44 patients with venous insufficiency compared to 34 control limbs. Where pathological reflux was noted in the venous system, especially in limbs with ulcers, there were statistically significant differences $(p<0.05)$ in range of ankle plantar flexion and strength of dorsiflexion $(p<0.05)$.

Findings from these studies suggest that the severity of venous insufficiency is correlated with restricted AROM, however, some loss of AROM exists in all clinical stages of chronic venous disorders. It is unclear whether venous insufficiency is caused, in part, by reduced AROM, or if AROM restricts calf muscle pump function. Nonetheless, these findings underscore the need to address impaired AROM when assessing patients for venous insufficiency or leg ulcer treatment, which, is currently not a standard assessment parameter for patients with venous disorders or leg ulcers.

\subsection{AROM and VRT}

VRT provides information about the overall function of the venous system. Faster refill time ( $\leq 25$ seconds) is associated with impaired venous blood flow; $<10$ seconds is associated with severe insufficiency. We found no statistically significant differences in AROM (eversion, inversion, dorsiflexion, plantar flexion) and VRT (marginal, moderate, severe) between the two groups. The hypothesis that there would be no difference in AROM for VRT was supported. To our knowledge, there are no studies that address the relationship between AROM and VRT. We found taking VRT measurements with the 
handheld PPG difficult in many subjects. In the presence of severe lipodermatosclerosis (hardening of the skin), the sensor would often not stick to the skin and in highly pigmented skin, errors in readings were encountered as the Doppler "signal" could not penetrate through the pigments. Thus, there is reason to question the validity of taking VRT measurements in patients with severe skin damage. The findings do not reveal useful data upon which to base clinical decisions.

\subsection{Strengths and Weaknesses}

We acknowledge the weaknesses of this study, including the small sample size and ability to control for multiple factors that may impact the physiologic outcomes such as co-morbid conditions. There was a lack of sufficient numbers of subjects in the different severity groups to truly analyze differences according to disease severity. Also, increased AC size $>25 \mathrm{~cm}$, obesity, and the presence of edema may restrict ankle motion, thus complicating the interpretation of the findings. However, the strength of this work has established that cooling inflamed skin did not pose a risk by further compromising AROM. Our results support findings from the literature that AROM is reduced in patients with chronic venous disorders, now a well-recognized phenomenon. When the ankle circumference is smaller, leg elevation may improve dorsiflexion, perhaps by spontaneous movements of the feet when the legs are at rest.

\subsection{Nursing Application}

To our knowledge, this is the first study to evaluate the effects of cooling inflamed skin associated with chronic venous disorders on AROM. Further clinical studies examining strategies for interventions with severely compromised AROM are needed. While studies show improvements in AROM after physical therapy and special exercises, feasible, sustainable, and novel treatments are needed for patients with severely deconditioned lower legs and ankles, obesity, and painful legs and ulcers to promote ankle mobility and physical activity. Without movement, skin damage and ulcers are slow to heal as the pumping action of the calf is necessary to reduce high venous pressures. In addition, studies are needed that examine the relationship between passive treatments to strengthen the ankle before a more strenuous active approach is taken, especially in patients with open ulcers being treated with high compression. The novel cooling gel wrap used in this study is currently being investigated in a larger randomized clinical trial conducted over a 9-month period. The aim is to determine if there is a reduction in the incidence of new or recurring leg ulcers during the study period. If this aim is achieved, cryotherapy could augment guideline-guided nursing care for the prevention of leg ulcers in patients with skin damage and a history of recurrent ulceration. Nurses who care for patients with venous disorders are uniquely positioned to assess the lower extremities for ankle range of motion. If reduced, physical activity recommendations through physical therapy and minimally exertive exercises could improve the strength and integrity of the ankle to promote pumping of the calf.

\section{ACKNOWLEDGEMENTS}

The project described was supported by Grant Number 010604 from the National Institute of Nursing Research. Supplemental support was provided by Award Number UL1RR029882 from the National Center for Research Resources. The content is solely the responsibility of the authors and does not necessarily represent the official views of the National Center for Research Resources or the National Institutes of Health.

\section{REFERENCES}

[1] Weingarten, M.S. (2001) Start-of-the-art treatment of chronic venous disease. Clinical Infection and Disease, 32, 949954. doi: $10.1086 / 319360$

[2] Herschthal, J. and Kirsner, R.S. (2008) Current management of venous ulcers: An evidence-based review. Surgical Technology International, 17, 77-83.

[3] Park, W., Ramachandran, J., Weisman, P. and Jung, E.S. (2010) Obesity effect on maleactive joint range of motion. Ergonomics, 53, 102-108. doi:10.1080/00140130903311617

[4] Simka, M. (2007) Calf muscle pump impairment and delayed healing of venous legulcers: Air plethysmographic findings. Journal of Dermatology, 34, 537-544. doi:10.1111/j.1346-8138.2007.00327.x

[5] Valencia, I.C., Falabella, A., Kirsner, R.S. and Eaglstein, W.H. (2001) Chronic venous in sufficiency and venous leg ulceration. Journal of the American Academy of Dermatology, 44, 401-412. doi:10.1067/mjd.2001.111633

[6] Back, T.L., Padberg, F.T., Araki, C.T., Thompson, P.N. and Hobson, R.W. (1995) Limited range of motion is a significant factor in venous ulceration. Journal of Vascular Surgery, 22, 519-523. doi:10.1016/S0741-5214(95)70030-7

[7] Ruckley, C.V., Dale, J.J., Callam, M.J. and Harper, D.R. (1982) Causes of chronic legulcer. Lancet, 2, 615-616. doi:10.1016/S0140-6736(82)90699-7

[8] Padberg, F.T., Johnston, M.V. and Sisto, S.A. (2004) Structured exercise improves calfmuscle pump function in chronic venous insufficiency: A randomized trial. Journal of Vascular Surgery, 39, 79-87. doi:10.1016/i.jvs.2003.09.036

[9] Yang, D., Vandongen, Y.K. and Stacey, M.C. (1999) Effect of exercise on calf musclepump function in patients with chronic venous disease. British Journal of Surgery, 86, 338-341. doi:10.1046/j.1365-2168.1999.00993.x

[10] Cavalheri, G., de Godoy, J.M.P. and Belczak, C.E.Q. (2008) 
Correlation of haemodynamics and ankle mobility with clinical classes of clinical, aetiologial, anatomical and pathological classification in venous disease. Phlebology, 23, 122-125. doi:10.1258/phleb.2007.007039

[11] Dix, F.P., Brooke, R. and McCollum, C.N. (2003) Venous disease is associated with animpaired range of ankle movement. European Journal of Vascular and Endovascular Surgery, 25, 556-561. doi:10.1053/ejvs.2002.1885

[12] Panny, M., Ammer, K., Kundi, M., Katzenschlager, R. and Hirschl, M. (2009) Severity of chronic venous disorders and its relationship to the calf muscle pump. VASA, 38, 171-176.

[13] Van Rij, A.M., De Alwis, C.S., Jiang, P., Christie, R.A., Hill, G.B., Dutton, S.J. and Thomson, I.A. (2008) Obesity and impaired venous function. European Journal of Vascular and Endovascular Surgery, 35, 739-744. doi:10.1016/j.ejvs.2008.01.006

[14] Willenberg, T., Schumacher, A., Amann-Vesti, B., Jacomella, V., Thalhammer, C., Diehm, N., Baumgartner, I. and Husmann, M. (2010) Impact of obesity on venous hemodynamics of the lower limbs. Journal of Vascular Surgery, 52, 664-668. doi:10.1016/i.jvs.2010.04.023

[15] Rao, S.R., Saltzman, C.L., Wilken, J. and Yak, H.J. (2006) Increased passive ankle stiffness and reduced dorsiflexion range of motion in individuals with diabetes mellitus. Foot and Ankle International, 27, 617-622.

[16] Margolis, D.J., Knauss, J. and Bilker, W. (2004) Medical conditions associated with venous leg ulcers. British Journal of Dermatology, 150, 267-273. doi:10.1111/j.1365-2133.2004.05773.x

[17] Seitz, C.S., Berens, N., Brocker, E.B. and Trautmann, A. (2010) Leg ulceration in rheumatoid arthritis-An underreported multicausal complication with considerable morbidity: Analysis of thirty-six patients and review of the literature. Dermatology, 220, 268-273. doi: $10.1159 / 000284583$

[18] Raju, S., Hollis, K. and Neglen, P. (2007) Use of compression stockings in chronic venous disease: Patient compliance and efficacy. Annals of Vascular Surgery, 21, 790795. doi:10.1016/j.avsg.2007.07.014

[19] Closs, S.J., Nelson, E.A. and Briggs, M. (2008) Can venous and arterial leg ulcers be differentiated by the characteristics of the pain they produce? Journal of Clinical Nursing, 17, 637-645. doi:10.1111/j.1365-2702.2007.02034.x

[20] Kelechi, T.J., Mueller, M., Zapka, J.G. and King, D.E.
(2011) The effect of a cryotherapy gel wrap on the microcirculation of the skin affected by chronic venous disorders. Journal of Advanced Nursing, 67, 2337-2349. doi:10.1111/j.1365-2648.2011.05680.x

[21] Collins, N.C. (2008) Is ice right? Does cryotherapy improve outcome for acute soft tissue injury? Emergency Medical Journal, 25, 65-68. doi:10.1136/emj.2007.051664

[22] Eklof, B., Rutherford, R.B, Bergan, J.J., Carpentier, P.H., Gloviczki, P., Kistner, R.L., Meissner, M.H., Moneta, G.L., Myers, K., Padberg, F.T., Perrin, M., Ruckley, C.V., Smith, P.C. and Wakefield, T.W. (2004) Revision of the CEAP classification for chronic venous disorders: Consensus statement. Journal of Vascular Surgery, 40, 1248-1252. doi:10.1016/j.jvs.2004.09.027

[23] Norkin, C. and White, D.J. (2009) Measurement of joint motion: A guide to goniometry. F. A. Davis Company, Philadelphia, 288-293.

[24] Youdas, J.W., Bogard, C.L. and Suman, V.J. (1993) Reliability of goniometric measurements and visual estimates of ankle joint active range of motion obtained in a clinical setting. Archives of Physical Medicine and Rehabilitation, 74, 1113-1118. doi:10.1016/0003-9993(93)90071-H

[25] Pieper, B., Templin, T.N., Birk, T.J. and Kirsner, R.S. (2007) Effects of injection-drug injury on ankle mobility and chronic venous disorders. Journal of Nursing Scholarship, 39, 312-318. doi:10.1111/j.1547-5069.2007.00186.x

[26] Eberhardt, R.T. and Raffetto, J.D. (2005) Chronic venous insufficiency. Circulation, 111, 2398-2409. doi:10.1161/01.CIR.0000164199.72440.08

[27] Whiteley, M.S., Horrocks, M. and Fox, A.D. (1998) Photoplethysmography can replace hand-held Doppler in the measurement of ankle/brachial indices. Annals of the Royal College of Surgeons of England, 80, 96-98.

[28] Sepic, S.B., Murray, M.P., Mollinger, L.A., Spurr, G.B. and Gardner, G.M. (1986) Strength and range of motion in the ankle in two age groups of men and women. American Journal of Physical Medicine, 65, 75-84.

[29] Grimston, S.K., Nigg, B.M., Hanley, D.A. and Engsberg, J.R. (1993) Differences in ankle joint complex range of motion as a function of age. Foot and Ankle, 14, 215-222.

[30] Lai, P.P., Leung, A.K., Li, A.N. and Zhang, M. (2008) Three-dimensional gait analysis of obese adults. Clinical Biomechanics, 23, S2-S6. doi:10.1016/j.clinbiomech.2008.02.004 